Chemische Thermodynamik 



\section{Chemische Thermodynamik}

von

Arnold Münster

Dr. rer. nat., o. Professor und Direktor

des Instituts für theoretische physikalische Chemie an der Universität Frankfurt/Main

Mit 34 Abbildungen

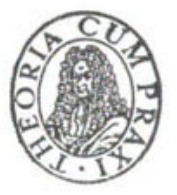

A K A D E M I E - V E R L A G - B E R L I N 1969 


\section{Lizenzausgabe \\ des Verlages Chemie, GmbH, Weinheim/Bergstr.}

(C) 1969 Verlag Chemie, GmbH, Weinheim/Bergstr.

Alle Rechte, insbesondere die der Ubersetzung in fremde sprachen, vorbehalten. Kein Teil dieses Buches darf ohne schriftliche Genehmigung des Verlages in irgendeiner Form - durch Photokopie, Mikrofilm oder irgendein anderes Verfahren - reproduziert oder in eine von Maschinen, insbesondere von Datenverarbeitungsmaschinen, verwendbare Sprache ïbertragen oder übersetzt werden. - All rights reserved (including those of translation into foreign languages). No part of this book may be reproduced in any form - by photoprint, microfilm, or any other means - nor transmitted, nor translated into a machine language without the permission in writing of the publishers.

Die Wiedergabe von Gebrauchsnamen, Handelsnamen, Warenbezeichnungen und dergl, in diesem Buch berechtigt nicht zu der Annahme, daß solche Namen ohne weiteres von jedermann benutzt werden dürfen. Vielmehr handelt es sich häufig um gesetzlich geschützte, eingetragene Warenzeichen, auch wenn sie nicht eigens gekennzeichnet sind.

Erschienen im A kademie-Verlag GmbH, 108 Berlin, Leipziger Straße 3-4

Lizenznummer: $202 \cdot 100 / 541 / 69$

Gesamtherstellung: VEB Druckerei ,Thomas Müntzer“, 582 Bad Langensalza

Bestellnummer: $5733 \cdot$ ES $18 \mathrm{C} 2$

Schutzumschlag und Einband: Karl Salzbrunn, Berlin 